\title{
Four-valued modal logic: Kripke semantics and duality*
}

\author{
Achim Jung and Umberto Rivieccio \\ School of Computer Science \\ University of Birmingham \\ Edgbaston, B15 2TT Birmingham \\ United Kingdom \\ A.Jung@cs.bham.ac.uk U.Rivieccio@cs.bham.ac.uk
}

Combining multi-valued and modal logics into a single system is a long-standing concern in mathematical logic and computer science, see for example [7] and the literature cited there. Recent work in this trend [15, 17, 14] develops modal expansions of many-valued systems that are also inconsistencytolerant, along the tradition initiated by Belnap with his "useful four-valued logic" [3]. Our contribution continues on this line, and the specific problem we address is that of defining and axiomatizing the least modal logic over the four-element Belnap lattice. The problem was inspired by [5], but our solution is quite different from (and in some respects more satisfactory than) that of [5] in that we make an extensive and profitable use of algebraic and topological techniques. In fact, our algebraic and topological analyses of the logic have, in our opinion, an independent interest and contribute to the appeal of our approach.

Kripke frames provide a semantics for modal logics that is both flexible with regards to intended applications and interpretations, and highly intuitive. When the non-modal part is multi-valued, though, one may wonder whether the accessibility relation between worlds should remain two-valued or be allowed to assume the same range of truth values as the logic itself. Starting from the point of view of AI applications, [7] argues forcefully that multiple values are an appropriate and useful modeling device. This is the approach taken in [5] and here, too. Our aim is to study the least modal logic over the Belnap lattice, that is, the logic determined by the class of all Kripke frames where the accessibility relation as well as semantic valuations are four-valued.

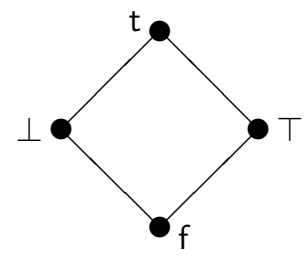

Figure 1: The four-element Belnap lattice FOUR

The non-modal system we build on is the propositional logic determined by the Belnap lattice shown in Figure 1, but the logical/algebraic language that we consider is more expressive than the one originally introduced by Belnap. We have not only conjunction, disjunction and negation $(\wedge, \vee, \neg)$ but also two implication operations, a weak $(\supset)$ and a strong one $(\rightarrow)$. Weak implication is defined by

$$
x \supset y:= \begin{cases}\mathrm{t} & \text { if } x \notin\{\mathrm{t}, \top\} \\ y & \text { if } x \in\{\mathrm{t}, \top\}\end{cases}
$$

${ }^{*}$ Submission to the conference on Topology, Algebra and Categories in Logic (TACL 2013), to be held in Nashville, Tennessee (USA), on July 28 - August 1, 2013. The corresponding author is the second one. 
while strong implication is given by the term $x \rightarrow y:=(x \supset y) \wedge(\neg y \supset \neg x)$. The set of designated elements of the logic (truth-like elements to be preserved in derivations) is the same as Belnap logic, namely $\{\mathrm{t}, \top\}$. We include these two elements as constants in the language, mainly for technical reasons (see below). The resulting logic in the language $\langle\wedge, \vee, \supset, \rightarrow, \neg, \mathrm{t}, \top\rangle$ can be viewed either as a fragment of the bilattice logic of Arieli and Avron [2] or as a language expansion (and an axiomatic extension) of paraconsistent Nelson logic [1, 11]. The second point of view is more suitable for our purpose as it allows us to apply known results (alongside new ones, proven here for the first time) on paraconsistent Nelson logic and its algebraic counterpart by simply specializing them to our case. Using the language of logical matrices [8], we may just say that our non-modal base logic is the propositional logic in the language $\langle\wedge, \vee, \supset, \rightarrow, \neg, \mathrm{t}, \top\rangle$ determined by the matrix $\langle F O U R,\{\mathrm{t}, \top\}\rangle$. A Hilbert-style axiomatization for this logic can be easily derived from the one introduced by Odintsov [11] for paraconsistent Nelson logic; in fact it is easy to prove that our logic is algebraizable [4] and its algebraic semantics is the variety generated by FOUR in the above-mentioned algebraic language.

For a modal expansion of this logic we initially focus on the necessity operator $\square$. Semantically, we interpret it in suitable Kripke structures. For motivation, let us consider first a classical Kripke model $\langle W, R, v\rangle$, where $W$ is a set of "worlds", $R$ an accessibility relation among them and $v$ a valuation. We view $R$ as the characteristic function associated with the accessibility relation, i.e., as a map $R: W \times W \rightarrow$ $\{0,1\}$. Similarly, view $v: F m \times W \rightarrow\{0,1\}$ as a map assigning to each formula $\varphi \in F m$ at each point $w \in W$ a truth value in $\{0,1\}$. By so-called standard translation of modal logic into first-order logic, we obtain the following definition for the semantics of the necessity operator

$$
v(\square \varphi, w):=\bigwedge\left\{R\left(w, w^{\prime}\right) \rightarrow v\left(\varphi, w^{\prime}\right): w^{\prime} \in W\right\}
$$

where $\wedge$ denotes the infinitary meet corresponding to the universal quantifier and $\rightarrow$ is Boolean implication. This definition can now easily be adapted to our four-valued setting. We consider Kripke models $\langle W, R, v\rangle$ where both $R$ and $v$ are four-valued, that is, we define $R: W \times W \rightarrow F O U R$ and $v: F m \times W \rightarrow F O U R$. As before, valuations are required to be homomorphisms in their first argument. Since FOUR carries two implications, there are at least two candidates for the translations of (1) into the four-valued setting, namely the pairs $\langle\wedge, \rightarrow\rangle$ and $\langle\wedge, \supset\rangle$. The latter option, which has been used in [15] to introduce a modal expansion of Belnap logic, has the disadvantage (in our opinion) that the accessibility relation $R$, although formally introduced as four-valued, has a two-valued behaviour when interacting with weak implication. This is so because in FOUR the value of (1), with $\rightarrow$ replaced by $\supset$, is the same as the following:

$$
\bigwedge\left\{v\left(\varphi, w^{\prime}\right): R\left(w, w^{\prime}\right) \in\{\mathrm{t}, \top\}\right\} .
$$

Our choice for the semantics of the $\square$ operator is thus based on the pair $\langle\wedge, \rightarrow\rangle$, that is, in the fourvalued context we replace classical conjunction with the lattice meet and classical implication with strong implication of FOUR. An important technical advantage of our choice is that FOUR can be viewed as a residuated lattice [9] with residuated pair $\langle *, \rightarrow\rangle$, the monoid operation $*$ being defined by the term $x * y:=\neg(y \rightarrow \neg x)$. We obtain thus a commutative monoid $\langle F O U R, *, \top\rangle$ satisfying the following property (residuation): $x * y \leq z$ iff $y \leq x \rightarrow z$ [16, Proposition 5.4.1]. This means that certain results of [5] can be straightforwardly transferred to our setting. For instance, given that strong implication interacts well with negation, we can define a possibility operator $\diamond$ as follows [5, p.746]:

$$
v(\diamond \varphi, w):=\bigvee\left\{R\left(w, w^{\prime}\right) * v\left(\varphi, w^{\prime}\right): w^{\prime} \in W\right\}
$$

This is obviously a generalization of the classical definition, with the monoid operation replacing classical conjunction; $\diamond$ and $\square$ are thus duals of one another, i.e., $\diamond \varphi$ is semantically equivalent to $\neg \square \neg \varphi$. The semantic definition of modal consequence is now straightforwardly obtained by adapting the classical one. We say that a point $w \in W$ of a four-valued Kripke model $M=\langle W, R, v\rangle$ satisfies a formula 
$\varphi \in F m$ if $v(\varphi, w) \in\{\mathrm{t}, \top\}$, and we write $M, w \vDash \varphi$. For a set $\Gamma \subseteq F m$, we write $M, w \vDash \Gamma$ to mean that $M, w \vDash \gamma$ for each $\gamma \in \Gamma$. As usual in modal logic, we consider two consequence relations. The local consequence $\Gamma \vDash_{l} \varphi$ holds if for every model $M=\langle W, R, v\rangle$ and every $w \in W$, it is the case that $M, w \vDash \Gamma$ implies $M, w \vDash \varphi$. The global consequence relation $\Gamma \vDash_{g} \varphi$ holds if, for every model $M$, if $M, w \vDash \Gamma$ for all $w \in W$, then $M, w \vDash \varphi$ for all $w \in W$.

Our main result is the introduction of two Hilbert-style calculi that provide complete axiomatizations for, respectively, $\vDash_{l}$ and $\vDash_{g}$ defined above. In this respect, we achieve a more satisfactory solution than [5], because the problem of axiomatizing the global consequence (even in the simplest case, the logic of one finite integral residuated lattice) is left open in [5].

The calculi we introduce are inspired by [5, Definition 4.6] and, as in [5], axioms of a rather technical nature involving the truth constants play a crucial role. An example is the following:

$$
\sim \diamond \varphi \rightarrow \sim \diamond \neg \sim \varphi
$$

where $\diamond \varphi:=\neg \square \neg \varphi$ and $\sim \varphi:=\varphi \supset \neg$ t. As in classical modal logic, the local and the global calculus share the same axioms, which in our case include schemata such as $\square$ t and $(\square \varphi \wedge \square \psi) \rightarrow \square(\varphi \wedge \psi)$. However, the formulas $\square \top$ and $\square(\varphi \rightarrow \psi) \rightarrow(\square \varphi \rightarrow \square \psi)$ are not valid: ours are non-normal modal logics. But again as in the classical case, the only difference between the local and the global calculus is that so-called monotonicity is only a rule of the global one: $\varphi \rightarrow \psi \vdash_{g} \square \varphi \rightarrow \square \psi$, but not of the local: $\varphi \rightarrow \psi \nvdash_{l} \square \varphi \rightarrow \square \psi$. The only rule of $\vdash_{l}$ is that of the non-modal fragment of the logic, modus ponens.

Given our proposed axiomatizations, the main steps we needed to prove completeness are the following.

I. Using standard algebraic logic techniques, we develop algebraic semantics corresponding to our syntactic calculi. This is straightforward in the case of $\vdash_{g}$ as monotonicity rule ensures (the non-modal fragment being itself algebraizable) that $\vdash_{g}$ is algebraizable. A more delicate passage is to show that, as in classical modal logic, the two calculi share the same algebraic counterpart. The local calculus is thus also complete (although in a weaker sense, again as in the classical setting) with respect to the same class of algebras, which is a variety of N4-lattices [11,12] with a modal operator that preserves finite meets, related to the BK-lattices of [14].

II. We look for a convenient representation of our algebraic semantics. As happens with other algebras of many valued logic (N4-lattices, BK-lattices, bilattices [6]), each algebra in our variety can be represented as a subalgebra (called a twist-structure) of a special square of some simpler algebra. For instance, each BK-lattice $\mathbf{A}$ can be viewed as a subalgebra of a special square of a modal Boolean algebra $\mathbf{B}$. Each algebraic operation of $\mathbf{A}$ is represented component-wise using operations of $\mathbf{B}$. In particular, for $A \subseteq B \times B$, the modal operator $\square_{A}$ of $\mathbf{A}$ is given by $\square_{A}\langle x, y\rangle=\left\langle\square_{B} x, \diamond_{B} y\right\rangle$, where $\square_{B}, \diamond_{B}$ are the modal operators (duals to one another) of $\mathbf{B}$. Similarly, in our case, each algebra $\mathbf{A}$ in our variety can be viewed as a subalgebra of a special square of a bimodal Boolean algebra $\mathbf{B}=\left\langle B, \sqcap, \sqcup,^{\prime}, \square_{B}^{1}, \square_{B}^{2}\right\rangle$, i.e., a Boolean algebra $\left\langle B, \sqcap, \sqcup,{ }^{\prime}\right\rangle$ that carries two independent finite meet-preserving operators $\square_{B}^{1}, \square_{B}^{2}$ (together with the respective duals $\diamond_{B}^{1}, \diamond_{B}^{2}$ ). The representation of the modal operator $\square_{A}$ is a generalization of [14], as we have $\square_{A}\langle x, y\rangle=\left\langle\square_{B}^{1} x \sqcap \square_{B}^{2}\left(y^{\prime}\right), \diamond_{B}^{1} y\right\rangle$. Our proof of this representation result uses crucially the constants $t, T$ and this is the main reason for including them in the logical signature. The twist-structure representation is quite powerful, and it can be extended to an equivalence between two naturally associated algebraic categories. In fact, even if different algebras $\mathbf{A}_{1}, \mathbf{A}_{2}$ can be subalgebras of the square of the same bimodal Boolean algebra $\mathbf{B}$, there is a one-to-one correspondence between each algebra $\mathbf{A}$ in our variety and triples $(\mathbf{B}, \nabla, \Delta)$ where $\mathbf{B}$ is a bimodal Boolean algebra and $\nabla, \Delta$ are, respectively, a filter and an ideal of $B$ which encode the information that is needed to uniquely determine A (see [12, 14]).

III. We develop a topological duality for bimodal Boolean algebras, drawing mainly on JónssonTarski duality for modal algebras. This is relatively straightforward, as to a modal algebra $\mathbf{B}$ corresponds 
a Stone space $\langle X(\mathbf{B}), \tau\rangle$ endowed with a relation $R$ that is used to represent the modal operators. In the same way, a bimodal Boolean algebra $\mathbf{B}$ gives rise to a bimodal Stone space $\left\langle X(\mathbf{B}), \tau, R_{1}, R_{2}\right\rangle$ where each of the two relations $R_{1}, R_{2}$ corresponds to one of the two independent operators of $\mathbf{B}$. However, we are not just interested in bimodal Boolean algebras, but in triples $(\mathbf{B}, \nabla, \Delta)$. We thus need to take care of the subsets $\nabla$ and $\Delta$, which are represented on the dual space by, respectively, a closed set and an open set $C(\nabla), O(\Delta) \subseteq X(\mathbf{B})$. This is the most technically delicate point, and we note that the presence of these subsets establishes a link between $R_{1}$ and $R_{2}$, for instance it holds that $p \in C(\nabla)$ and $\langle p, q\rangle \in R_{2}$ imply $\langle p, q\rangle \in R_{1}$ for all $p, q \in X(\mathbf{B})$.

IV. We join the results of the previous items in order to build our proof of completeness. We assume, by contradiction, that our calculus (either the global or the local one, the reasoning is essentially the same) does not prove $\Gamma \vdash \varphi$. Then, by algebraic completeness (I), we can find an algebraic countermodel, that is, an algebra $\mathbf{A}$ together with a set of designated elements $F \subseteq A$ (a lattice filter with some additional properties) and a valuation $h: F m \rightarrow \mathbf{A}$ (a homomorphism from the algebra of formulas into A) such that $h(\gamma) \in F$ for all $\gamma \in \Gamma$ but $h(\varphi) \notin F$. By (II) we know that $\mathbf{A}$ can be represented as a twist-structure over a bimodal Boolean algebra $\mathbf{B}$, but it is essential here that we have a way of decomposing not only A but also $F$ and $h$ component-wise, thus obtaining a different algebraic countermodel based on $\mathbf{B}$, which is easier to handle. This is done essentially by taking projections, so we obtain two valuations $h_{1}$ and $h_{2}$ (the second projection of $F$ does not actually play any role due to the particular properties of this lattice filter). Now we use (III) to obtain a topological counter-model, in the same way as in classical logic (using the ultrafilter theorem). Finally, we show that a topological counter-model based on a bimodal Stone space $\left\langle X(\mathbf{B}), \tau, R_{1}, R_{2}\right\rangle$ can be turned into a four-valued Kripke model of the type introduced when we defined our Kripke semantics. This is relatively easy, as we can take $X(\mathbf{B})$ itself as the set of points and, in order to define a four-valued relation $R$, we let $R(p, q)=\mathrm{t}$ iff $\langle p, q\rangle \in R_{1} \cap R_{2}, R(p, q)=\top$ iff $\langle p, q\rangle \in R_{1} / R_{2}$, etc. for all $p, q \in X(\mathbf{B})$. In this way we have a four-valued Kripke frame $\langle X(\mathbf{B}), R\rangle$ and we can compose the above-mentioned valuations $h_{1}$ and $h_{2}$ with the embedding of $\mathbf{B}$ into the algebra of clopens of $X(\mathbf{B})$ given by Stone duality, to define a pair of two-valued valuations $v_{1}, v_{2}: F m \times X(\mathbf{B}) \rightarrow\{1,0\}$. These are turned into one four-valued valuation $v: F m \times X(\mathbf{B}) \rightarrow\{F O U R\}$ in the way to be expected, i.e., we define $v(\psi, p):=\mathrm{t}$ iff $v_{1}(\psi, p)=1$ and $v_{2}(\psi, p)=0, v(\psi, p):=\top$ iff $v_{1}(\psi, p)=v_{2}(\psi, p)=1$, etc. The twist-structure construction guarantees that $v$ acts homomorphically at the points of $X(\mathbf{B})$. We thus have a Truth Lemma that allows us to conclude that $\langle X(\mathbf{B}), R, v\rangle$ is indeed a four-valued Kripke model. This is the counter-model we were looking for to establish that $\Gamma \not \forall \varphi$, so our proof is finished.

Let us conclude by discussing the role played by constants. We presented in [10] a result which can now be viewed as a special case of the above, the main differences being that in [10] the non-modal base logic was the bilattice logic of [2] in which, moreover, all constants $(t, \top, f, \perp)$ were included in the language. As a consequence, the axiomatization of [10] can be easily obtained (at least for the local consequence) from that of [5], and the algebraic/topological analysis involved in the completeness proof is also more straightforward. It seems, semantically speaking, that the increased expressivity gained thanks to the constants is needed to restrict and classify the algebraic and topological models of the logic. In fact, [5] requires the presence in the language of all constants (one for each element of a fixed finite lattice), and it is interesting to note that the completeness proofs of [5] are based on a completely different strategy from ours.

However, the present work shows that in the case of FOUR we do not need all the constants, as we can dispense with $\perp$. This slight generalization is all but trivial, as it involves conjecturing and interpreting new axioms (such as (2) above) whose meaning is only clarified by a close analysis of the algebraic and topological models. We do not know whether it is possible (in the case of FOUR or, more in general, of a finite lattice) to obtain a method for proving completeness that does not require any constant. In our case, the main difficulty lies in steps (II)-(III). In the absence of constants, in fact, 
we may still be able to obtain an algebraic counter-model, which can then be turned into a topological one by applying Jónsson-Tarski duality together with the duality for N4-lattices introduced by Odintsov [13]. However, such model will be a topological space $X$ endowed with a two-valued relation $R$. In order to turn this structure into a four-valued Kripke model (thus concluding our completeness proof), we would need to view $R$ as a four-valued relation (or, equivalently, as a pair of relations $R_{1}, R_{2}$ ) defined on (a subset of) $X$ as described in [10]. This is the crucial step, which somehow corresponds to the twist-structure representation (II) on the algebraic side. Unfortunately, for the time being it seems that only using (some) constants we can express properties which ensure that, algebraically, models are representable as twist-structures and, on the topological side, that the two-valued relation $R$ can be viewed as a four-valued one.

\section{References}

[1] A. Almukdad and D. Nelson. Constructible falsity and inexact predicates. The Journal of Symbolic Logic, 49(1):231-233, 1984.

[2] O. Arieli and A. Avron. Reasoning with logical bilattices. Journal of Logic, Language and Information, 5(1):25-63, 1996.

[3] N. D. Belnap, Jr. A useful four-valued logic. In J. M. Dunn and G. Epstein, editors, Modern uses of multiplevalued logic (Fifth Internat. Sympos., Indiana Univ., Bloomington, Ind., 1975), pages 5-37. Episteme, Vol. 2. Reidel, Dordrecht, 1977.

[4] W. J. Blok and D. Pigozzi. Algebraizable logics, volume 396 of Mem. Amer. Math. Soc. A.M.S., Providence, January 1989.

[5] F. Bou, F. Esteva, L. Godo, and R. Rodríguez. On the minimum many-valued modal logic over a finite residuated lattice. Journal of Logic and Computation, 21(5):739-790, 2011.

[6] F. Bou, R. Jansana, and U. Rivieccio. Varieties of interlaced bilattices. Algebra Universalis, 66(1):115-141, 2011.

[7] M. Fitting, Many-valued modal logics. Fundamenta Informaticae, 15:235-254, 1991.

[8] J. M. Font and R. Jansana. A general algebraic semantics for sentential logics, of Lecture Notes in Logic, 7. Springer-Verlag, second edition, 2009.

[9] N. Galatos, P. Jipsen, T. Kowalski, and H. Ono. Residuated Lattices: an algebraic glimpse at substructural logics, volume 151 of Studies in Logic and the Foundations of Mathematics. Elsevier, Amsterdam, 2007.

[10] A. Jung and U. Rivieccio. Kripke semantics for modal bilattice logic (extended abstract). Proceedings of LICS 2013, accepted.

[11] S. P. Odintsov. Algebraic semantics for paraconsistent Nelson's logic. Journal of Logic and Computation, 13(4):453-468, 2003.

[12] S. P. Odintsov. On the representation of N4-lattices. Studia Logica, 76(3):385-405, 2004.

[13] S. P. Odintsov. Priestley duality for paraconsistent Nelson's logic. Studia Logica, 96(1):65-93, 2010.

[14] S. P. Odintsov and E. I. Latkin. BK-lattices. Algebraic semantics for Belnapian modal logics. Studia Logica, 100(1-2):319-338, 2012.

[15] S. P. Odintsov and H. Wansing. Modal logics with Belnapian truth values. Journal of Applied Non-Classical Logics, 20:279-301, 2010.

[16] U. Rivieccio. An Algebraic Study of Bilattice-based Logics. Ph. D. Dissertation, University of Barcelona, 2010.

[17] U. Rivieccio. Paraconsistent modal logics. Electronic Notes in Theoretical Computer Science, 278:173-186, 2011. 